\title{
Análise da situação do enfermeiro em um centro de materiais e esterilização: Revisão de literatura
}

Analysis of the nurse's situation in a materials and sterilization center: Literature review

Análisis de la situación de la enfermera en un centro de materiales y esterilización: Revisión de la literature

Recebido: 04/03/2021 | Revisado: 11/03/2021 | Aceito: 18/06/2021 | Publicado: 01/07/2021

Lya Carla Diniz Dos Anjos

ORCID: https://orcid.org/0000-0001-5897-1465

Faculdade Gianna Beretta, Brasil

E-mail: lya_carl1@hotmail.com

José Eduardo Batista

ORCID: https://orcid.org/0000-0003-2451-4789

Universidade Federal do Maranhão, Brasil

E-mail: jbatistaufma@gmail.com

Clice Cunha Pimentel de Sousa

ORCID: https://orcid.org/0000-0002-0094-2423

Universidade Federal do Maranhão, Brasil

E-mail: clicecunha@hotmail.com

Franco Celso da Silva Gomes

ORCID: https://orcid.org/0000-0002-7381-924X

Universidade Federal do Maranhão, Brasil

E-mail: fcsilva-gomes@hotmail.com

Rachel de Jesus Pimentel Araújo

ORCID: https://orcid.org/0000-0002-1002-6293

Universidade Federal do Maranhão, Brasil

E-mail: rajepi_araujo@hotmail.com

Luzinete Pontes Brandão

ORCID: https://orcid.org/0000-0002-8592-5621

Faculdade Supremo Redentor, Brasil

E-mail: brandao.nethe@hotmail.com

Larissa Maria Gava dos Santos

ORCID: https://orcid.org/0000-0002-7078-941X

Faculdade São Leopoldo Mandic, Brasil

E-mail: lariigava@gmail.com

Maria do Socorro Marques Soares

ORCID: https://orcid.org/0000-0002-9734-4808

Universidade Federal do Maranhão, Brasil

E-mail: maria.soares@ifma.edu.br

Ana Patricia Fonseca Coelho Galvão

ORCID: https://orcid.org/0000-0003-3376-5678

Faculdade de Ciências Médicas da Santa Casa de São Paulo, Brasil

E-mail:ana.coelho@ceuma.br

Bruna Rayana Santos Cunha

ORCID: https://orcid.org/0000-0001-8177-5155

Universidade Ceuma, Brasil

E-mail: brunacunha2012@gmail.com

Alinne Silva Andrade Costa

ORCID: https://orcid.org/0000-0001-6628-2571

Secretária Municipal da Saúde, Brasil

E-mail: alinnesac@hotmail.com

Andrea Suzana Vieira Costa

ORCID: https://orcid.org/0000-0003-4490-766X

Universidade Federal do Maranhão, Brasil E-mail:andrea.vieira@ufma.br

Gersuinete Rodrigues Bastos dos Santos

ORCID: https://orcid.org/0000-0002-1602-4563

Universidade Federal do Maranhão, Brasil

E-mail: gerusinete@hotmail.com

Francisca Bruna Arruda Aragão

ORCID: https://orcid.org/0000-0002-1191-0988

Universidade de São Paulo, Brasil

E-mail: aragao_bruna@usp.br 


\begin{abstract}
Resumo
O Centro de Materiais e Esterilização (CME) configura um ambiente importante na prestação de cuidados em uma instituição de saúde e o Enfermeiro é um profissional fundamental no gerenciamento do processo de trabalho no referido setor. O estudo tem por objetivo averiguar a situação do Enfermeiro frente ao Centro de Materiais e Esterilização. Tratase de uma revisão de literatura, com levantamento de dados em livros, dissertações e artigos científicos através das bases de dados disponíveis na Biblioteca Virtual de Saúde: Literatura Latino-Americana do Caribe em Ciências da Saúde (LILACS) e Base de Dados Bibliográficos Especializada na Área de Enfermagem (BDENF); no portal de publicação cooperativa Scientific Electronic Library Online (SCIELO) e na plataforma de livros digitais do Google Books. Verificou-se, por meio das análises dos estudos, a importância do Centro de Materiais e Esterilização para o funcionamento de uma instituição de saúde e que seu funcionamento exige critérios que devem ser rigorosamente atendidos, conforme preconiza a Resolução de Diretoria Colegiada - RDC n. 15. Por ser um setor complexo e que exige cuidados adequados requer a atuação de um profissional de enfermagem capacitado para nele trabalhar. Embora os estudos apontam a importância do Enfermeiro no gerenciamento do CME, alguns indicam que a ausência do Enfermeiro ou desvalorização dos profissionais de enfermagem afetam a qualidade do serviço prestado, bem como a saúde desses trabalhadores. Considera-se a necessidade de mais estudos que evidenciam mais a situação do Enfermeiro em relação ao CME e que sugerem políticas de melhorias das condições de trabalho e valorização da equipe de enfermagem que atua nesse setor.
\end{abstract}

Palavras-chave: Centro de materiais e esterilização; Enfermagem; Trabalho; Enfermeiro.

\begin{abstract}
The Materials and Sterilization Center (CME) sets up an important environment in the provision of care in a health institution and the Nurse is a fundamental professional in the management of the work process in that sector. The study aims to ascertain the situation of the Nurse in front of the Materials and Sterilization Center. It is a literature review, with data collection in books, dissertations and scientific articles through the databases available in the Virtual Health Library: Latin American Caribbean Literature in Health Sciences (LILACS) and Bibliographic Database Specialized in the Nursing Area (BDENF); on the Scientific Electronic Library Online (SCIELO) cooperative publishing portal and on the Google Books digital book platform. Through the analysis of the studies, it was verified the importance of the Materials and Sterilization Center for the functioning of a health institution and that its operation requires criteria that must be strictly met, as recommended by the Collegiate Board Resolution - RDC n. 15. As it is a complex sector that requires adequate care, it requires the performance of a qualified nursing professional to work in it. Although the studies point out the importance of the nurse in the management of the CME, some indicate that the absence of the nurse or the devaluation of nursing professionals affect the quality of the service provided, as well as the health of these workers. It is considered the need for more studies that show more the situation of the Nurse in relation to the CME and that suggest policies for improving working conditions and valuing the nursing team that works in this sector.
\end{abstract}

Keywords: Materials and sterilization center; Nursing; Job; Nurse.

\title{
Resumen
}

El Centro de Materiales y Esterilización (CME) configura un entorno importante en la prestación de cuidados en una institución de salud y la Enfermera es un profesional fundamental en la gestión del proceso de trabajo en ese sector. El estudio tiene como objetivo conocer la situación de la enfermera frente al Centro de Materiales y Esterilización. Se trata de una revisión de literatura, con recolección de datos en libros, disertaciones y artículos científicos a través de las bases de datos disponibles en la Biblioteca Virtual en Salud: Literatura del Caribe Latinoamericano en Ciencias de la Salud (LILACS) y Base de Datos Bibliográfica Especializada en el Área de Enfermería (BDENF); en el portal de publicación cooperativa Scientific Electronic Library Online (SCIELO) y en la plataforma de libros digitales Google Books. Mediante el análisis de los estudios, se verificó la importancia del Centro de Materiales y Esterilización para el funcionamiento de una institución de salud y que su funcionamiento requiere de criterios que deben cumplirse estrictamente, tal como lo recomienda la Resolución del Consejo Colegiado - RDC n. 15. Al tratarse de un sector complejo y que requiere cuidados adecuados, requiere la actuación de un profesional de enfermería calificado para trabajar en él. Si bien los estudios señalan la importancia del Enfermero en la gestión del CME, algunos señalan que la ausencia del Enfermero o la desvalorización de los profesionales de enfermería afectan la calidad del servicio brindado, así como la salud de estos trabajadores. Se considera la necesidad de más estudios que muestren más la situación de la Enfermera en relación con el CME y que sugieran políticas para mejorar las condiciones laborales y valorar el equipo de enfermería que trabaja en este sector.

Palabras clave: Centro de materiales y esterilización; Enfermería; Trabajo; Enfermero.

\section{Introdução}

O Centro de Material e Esterilização (CME) constitui um setor fundamental de uma instituição de saúde, uma vez que dá grande suporte nos serviços hospitalares e colabora para o atendimento dos usuários através de uma atuação constituída por etapas internas de produção sendo, portanto, considerado um serviço de cuidados. 
A qualidade do processamento de trabalho no CME tem influência no dimensionamento do pessoal de enfermagem, tendo o Enfermeiro como agente fundamental no gerenciamento desse processo. Entretanto, seu trabalho vem imbuído de certa invisibilidade perante os demais setores das instituições de saúde e que requer um reconhecimento socioprofissional.

Essa problemática implica na necessidade de refletir e discutir, com os trabalhadores do CME, a construção de estratégias e intervenções sobre a invisibilidade do trabalho de enfermagem e os possíveis meios de romper ou minimizar o estereótipo da invisibilidade que se sobrepõe com a falta de reconhecimento e valorização dos processos de trabalho de enfermagem do Centro de Material e Esterilização (Silva, 2017).

A importância do Enfermeiro no CME e seus afazeres cruciais no controle de infecção hospitalares, na promoção de um atendimento eficiente e humanizado à população merecem visibilidade. Desse modo, o presente trabalho tem por objetivo averiguar a situação do Enfermeiro frente ao Centro de Materiais e Esterilização.

O estudo parte da necessidade de se discutir de forma incisiva o referido tema, visto que poucos estudos a nível de Brasil dão ênfase no trabalho do Enfermeiro no CME de forma positiva, sendo muitas das vezes desvalorizado por não ter visibilidade social e remuneração adequada. Devido processo de trabalho ser complexo, os profissionais de enfermagem estão sujeitos a eventuais riscos físicos e ocupacionais.

No decorrer do trabalho foi abordado alguns pontos importantes do CME e seu dimensionamento físico, processo de trabalho e principais riscos. Adiante, o estudo foca na discussão sobre a atuação da enfermagem no CME, carga de trabalho e valorização profissional, bem como o papel do Enfermeiro e sua importância no gerenciamento desse setor, além da educação continuada como estratégia de melhoramento profissional do Enfermeiro e de sua equipe.

\section{Metodologia}

O estudo é uma revisão de literatura, de pesquisa bibliográfica que é uma atividade de investigação em material teórico sobre o assunto de interesse. Por se tratar de um processo de levantamento e análise do problema de pesquisa escolhido, a pesquisa bibliográfica o mapeamento do que já foi escrito sobre o tema da pesquisa (Marconi \& Lakatos, 2018). Precede o reconhecimento do problema ou do questionamento que funciona como delimitador do problema de estudo.

Visando atender com rigor o processo metodológico, seguiram-se as seguintes etapas para a realização deste estudo: identificação de problema, com a definição da questão de pesquisa; estabelecimento de critérios para inclusão e exclusão de estudos para a busca de literatura científica; definição das informações a serem extraídas dos estudos; avaliação dos estudos; interpretação dos resultados e apresentação da revisão/síntese do conhecimento.

A busca dos dados foi realizada em bases de dados disponíveis na Biblioteca Virtual de Saúde, tais como: Literatura Latino-Americana do Caribe em Ciências da Saúde (LILACS) e Base de Dados Bibliográficos Especializada na Área de Enfermagem (BDENF); no portal de publicação cooperativa Scientific Electronic Library Online (SCIELO), além da plataforma de livros digitais do Google Books.

Na seleção dos conteúdos, foram adotados como critérios de inclusão: dissertação, livro e artigo original com texto completo disponível na íntegra gratuitamente, publicados no período de 2012 a 2020, disponibilizados no idioma português. Dissertações, livros e artigos incompletos e/ou que não atenderam ao período estabelecido não foram incluídas desta revisão.

A coleta de dados ocorreu no período de abril a junho de 2020. A amostragem dos dados resultou no total de 105 publicações, descarte 18 publicações com duplicata. A análise e extração dos dados decorreram-se através da leitura de títulos, resumos e artigos completos dos 87 estudos restantes. Após esse processo, 43 estudos atenderam aos critérios de elegibilidade e ao objetivo da pesquisa, completando, assim, a amostra da revisão. A seguir, foi realizada a leitura dos textos, fichamento e extração das informações para dinamizar o desenvolvimento do presente artigo. 


\section{O Serviço no Centro de Material e Esterilização em Unidades de Saúde}

O Centro de Material e Esterilização (CME) tem como histórico o acompanhamento de procedimentos cirúrgicos, tutelando melhores condições aos processos mais invasivos nos cuidados cirúrgicos e pós-cirúrgicos. Com o avanço das pesquisas em medicina e a organização dos serviços de saúde, houve maior exigência no preparo dos materiais e da rouparia para os procedimentos cirúrgicos. Assim, foi implantado um espaço próprio, com a instalação de equipamentos e designação de pessoas capacitadas para as tarefas de limpeza, preparo e acondicionamento, controle e distribuição desses materiais (Souza, 2012).

Para Fusco e Spiri (2014), os avanços das técnicas cirúrgicas e a responsabilidade pelo controle de infecções fazem com que o CME tenha destaque dentro do hospital. Todavia, para que se cumpra, são necessários investimentos em qualificação, pesquisa e integração com as unidades consumidoras responsáveis pelas tarefas de prevenção, diagnóstico e terapêutica aos seus clientes.

Moraes (2012) aponta algumas vantagens do CME, tais como: economia de material e pessoal, controle de gastos e suprimentos, controle adequado do material, pessoal treinado, possibilitando maior confiabilidade no processo de esterilização.

\subsection{CME: alguns conceitos}

O Centro ou a Central de Materiais e Esterilização (CME) compreende a uma unidade de apoio técnicos a todas as áreas do hospital, responsável pelo processamento dos artigos como instrumental, roupas e outros. Para Santos (2017), corresponde a um setor específico e próprio, independente do centro cirúrgico, cujo objetivo é cuidar de todo o material inerente à instituição, cirúrgico ou não.

Segundo o Ministério da Saúde, o CME é um conjunto de elementos destinados a expurgo, preparo e esterilização, guarda e distribuição de material para as unidades do hospital, vinculado ou não ao centro cirúrgico, estando em observâncias às determinações das normas técnicas NR-9 de programa de prevenção de riscos ambientais (Brasil, 2009).

Madeira et al. (2015) define o CME como uma unidade funcional destinada ao processamento de produtos para a saúde, cuja missão é abastecer os serviços assistenciais e de diagnóstico com materiais processados, garantindo quantidade e a qualidade necessárias para uma assistência segura.

\subsection{Parâmetros Espaciais e Físicos do CME}

No tocante a prestação de serviços do CME, é necessário que o setor seja de acordo com os padrões de dimensionamento e estrutura física preconizados pela Resolução da Diretoria Colegiada - RDC's n. 50 e 307 de 2002, respectivamente - que constitui de área de lavagem e descontaminação, área de preparo de materiais, área de esterilização, área de armazenamento e distribuição de materiais e roupas esterilizados, além de determinar quando houver centro cirúrgico, centro obstétrico e/ou ambulatorial, hemodinâmica, emergência de alta complexidade e urgência seja implantado um Centro de Material e Esterilização.

Vital et al. (2014) salienta que, no Brasil, grande parte das unidades de saúde, embora realizam limpeza dos materiais de forma adequada, nem todas apresentam um ambiente com as recomendações mínimas, em se tratando da estrutura e dimensão. Muitas possuem setor de CME diferente do preconizado, em termos de dimensão mínima, e sem os equipamentos necessários. Santos (2017) explana algumas áreas que compõem o CME, que são:

a) Recepção e expurgo: área que recebe material sujo, vindo do centro cirúrgico e de outras unidades de internação. É aonde se faz a limpeza, através de máquinas ou material destinado a esse processo. É necessário que o profissional use EPI, além da touca descartável. 
b) Preparo do material: após a lavagem, o material é passado para essa área para secagem e preparo de seu acondicionamento, sendo posteriormente encaminhado à esterilização. Os pacotes são embalados em material próprio, como tecido de algodão cru, papel grau cirúrgico ou caixas metálicas (ex. Cl.Cir.= pacote de curativo, UTI $\mathrm{NN}=$ band. de flebotomia, $\mathrm{CC}=$ caixa básica 1 ).

c) Áreas de esterilização: é o local reservado para a esterilização propriamente dita do material, por processo físico ou químico, cujo objetivo é destruir todas as formas de vida microbiana. Conta com equipamentos como as autoclaves a vapor.

d) Área de guarda e distribuição de materiais: é a área destinada ao armazenamento/acondicionamento dos materiais que já sofreram o processo de esterilização. É também a área responsável pela distribuição do material entre as demais unidades.

De acordo com explanação acima, compreende-se que o planejamento da área física do CME deve estar bem alinhado às atividades do processo de trabalho

\subsection{O processo de Trabalho no CME}

A Agência Nacional de Vigilância Sanitária (ANVISA), através da RDC n. 15 de 15 de março de 2012, aprovou o regulamento técnico que estabelece os requisitos e boas práticas para o processamento de produtos para a saúde. Tal Resolução determina que os artigos para saúde classificados como críticos devem ser submetidos ao processo de esterilização, após processo de limpeza, inspeção, secagem e empacotamento. Os artigos para a saúde classificados como semicríticos devem ser submetidos, no mínimo, ao processo de desinfecção de alto nível, após processo de limpeza (Brasil, 2012).

O processo de trabalho em Centro de Materiais e Esterilização consiste nas etapas de limpeza, preparo/montagem, esterilização, guarda e distribuição de materiais e instrumentais odonto-médico-hospitalares, garantindo o abastecimento de toda a instituição de saúde, cabendo à equipe de enfermagem o fornecimento de materiais íntegros e ausentes de contaminação (Rosa, Mimura \& Borges, 2019).

No tocante aos artigos utilizados na assistência ventilatória, anestesia e inaloterapia, a RDC n. 15 determina que os eles devem ser submetidos à limpeza e à desinfecção a nível intermediário, com produtos saneantes em conformidade com a normatização sanitária, ou por processo físico de termodesinfecção, antes da utilização em outro paciente (Brasil, 2012; Moraes, 2012).

Nas ideias de Martins e Antunes (2019), o processo de trabalho no CME se assemelha, em alguns aspectos, ao processo de produção industrial no que cerne a divisão do processo de trabalho e a maneira de execução das atividades. Com a introdução de equipamentos automatizados, esse processo vem se tornando mais sofisticado, exigindo um quadro de pessoal adequado em quantidade e qualidade.

Silva (2017) frisa que, além do cuidado com artigos e materiais de uso médico, há a responsabilidade de evitar eventos adversos associados a resíduos de material biológico de um paciente para outro através de materiais reprocessados ou de resíduos dos produtos químicos utilizados durante a limpeza dos artigos.

\subsubsection{Riscos físicos e ocupacionais}

No Centro de Materiais e Esterilização, os profissionais da saúde, em especial os de enfermagem são fundamentais no processo de trabalho de CME. Sabe-se da importância do Enfermeiro no gerenciamento desse setor. Por sua vez é notória a necessidade das rotinas, espaço e equipamentos adequados, valorização profissional, visto que o Enfermeiro e sua equipe estão expostos aos riscos no desenvolver de suas atividades laborais (Seibel, 2017). 
Convém citar alguns riscos que afetam o trabalho e a saúde do trabalhador de CME, que são: riscos físicos, acidentes que decorrem do ambiente físico e do processo de trabalho e condições tecnológicas, impróprias, capazes de provocar lesões à integridade física do trabalhador; os riscos químicos estão relacionados a substâncias químicas que se encontram nas formas líquida, sólida e gasosa; os riscos biológicos estão relacionados a vírus, bactérias e protozoário, fungos e bacilos e parasitas; os riscos ocupacionais podem estar relacionados ao uso inadequado de Equipamentos de Proteção Individual (EPIs), carga de trabalho e valorização do trabalhador (Aquino, 2014; Araruna \& Posso, 2014).

Nos riscos físicos podem ser detectados ruídos, vibrações, calor, radiações ionizantes, radiações não-ionizantes, umidade e frio. A precariedade do serviço relacionado a falta de materiais e instalações inadequadas implicam em sofrimento dos trabalhadores, pois os levam ao constrangimento ao realizar um trabalho de má qualidade (Fontana \& Lautert, 2013).

Nos riscos ocupacionais, o arejamento/ ventilação insuficientes são um dos fatores que causam desconforto no ambiente de trabalho. Incluem-se nesse tipo de risco os psicossociais que submetem os profissionais ao sofrimento e adoecimento físico e mental, oriundos de agressão verbal e falta de segurança (Lima et al., 2018). Os profissionais de enfermagem sentem-se sujeitos constantemente a cargas diversas e simultâneas no trabalho, desatenção e descuido enquanto se encontram nas dependências do CME (Bittencourt, 2015).

De acordo com Carvalho et al. (2019), os riscos ocupacionais observados em pesquisas nacionais, as lesões com perfuro cortantes e as queimaduras por autoclave foram as mais representativas nas instituições. Outros fatores mencionados pelos autores como riscos de incêndio, contato com substâncias químicas e biológicas, exposição a ruídos, esforço físico, queda de materiais, desconforto por postura adotada e sobrecarga de trabalho contribuem para o surgimento de danos à saúde, como varizes, problemas oculares, lombalgia, transtorno do sono e lesões na coluna.

Lima et al. (2018), salientam que a identificação de situações em que há exposição dos profissionais de Enfermagem, possibilitam a implementação de estratégias de intervenção à saúde para a melhoria da qualidade de vida do trabalhador. As recomendações da Norma Regulamentadora no 9 (NR-9) do Ministério do Trabalho e Emprego (MTE) visa a preservação da saúde e da integridade dos trabalhadores através da antecipação, reconhecimento, avaliação e consequente controle da ocorrência de riscos ambientais existentes ou que venham a existir no ambiente de trabalho (Borgheti, Viegas \& Caregnato, 2016).

Em observância às determinações da NR-9 algumas estratégias que visam boas condições de trabalho e saúde do trabalhador merecem destaque são os programas de promoção da saúde e melhoria da qualidade de vida, os quais incorporam a ginástica laboral como atividade promotora da saúde física e mental dos trabalhadores, educação continuada são indispensáveis e devem fazer parte da cultura das instituições (Costa et al., 2015).

As medidas de biossegurança protegem, eliminam ou minimizam os riscos existentes. É indispensável o uso do Equipamento de Proteção Individual compostos de luvas, máscaras, óculos, avental, gorro, capote e calçado fechado, que são essenciais na prevenção de acidentes e na proteção contra a contaminação, conferindo ao trabalhador maior segurança no seu ambiente laboral (Alves et al., 2017). De acordo com Mendes (2017), os EPI's devem ser ofertados de forma gratuita, conforme a Norma Regulamentadora do Ministério do Trabalho (NR), para garantir a segurança dos que estão expostos aos riscos de perfuração ou corte, prevenindo acidentes de trabalho ou doenças ocupacionais.

Além dos EPI's, Santos et al. (2017) defendem a necessidade de realização de uma educação continuada e permanente como estratégia das medidas preventivas no intuito de sensibilizar os profissionais quantos aos cuidados necessários visando evitar os acidentes em CME.

\section{A Atuação da Equipe de Enfermagem no Centro de Materiais e Esterilização}

O Centro de Material e Esterilização (CME) é uma unidade fundamental de uma instituição de saúde, fazendo parte da sua infraestrutura e colaborando para o bom atendimento dos usuários através de uma atuação constituída por etapas de produção 
interna. Por ser um serviço da área de saúde de suma complexidade, configura-se como um setor de cuidados. Sendo assim, formadores e estudantes da área da saúde devem-se atentar à importância do CME (Lucon et al., 2017). Abrange hospitais gerais, especializados, bem como unidades básicas de saúde (UBS), ambulatórios médicos especializados (AME), unidades de assistência médica ambulatorial (AMA) e unidades de pronto atendimento (UPA), ficando sob a responsabilidade da equipe enfermagem (Marcondes \& Montanari, 2020).

Devida a importância e particularidade do CME nas unidades de saúde bem como o profissional que nele atua, é imprescindível analisar o papel da enfermagem que atua nesse setor, se o CME está incluso significativamente na formação teórica e prática dos cursos de enfermagem, a importância do Enfermeiro que atua no CME, além da educação continuada como pilar na produção de conhecimento e capacitação profissional, focando no treinamento, aprimoramento profissional e melhorias no setor.

\subsection{Perfil do Enfermeiro que Atua no CME}

O CME é tratado como setor peculiar em comparação aos demais setores de uma unidade hospitalar, em especial, no tocante à atuação do Enfermeiro, visto que a execução de tarefas exige conhecimento científico e tecnológico para coordenar o trabalho, almejando uma comunicação efetiva com as unidades de apoio e consumidoras do hospital, focando na relação de reciprocidade (Bugs et al., 2017).

Padoveze et al. (2013) corroboram com as afirmações de Bugs et al. (2017) ao citarem que, mediante a complexidade e a responsabilidade frente a questões cruciais envolvendo a esterilização de artigos, existe a necessidade de que todas as etapas do processamento do CME devam ser supervisionadas por pessoa qualificada e competente, com conhecimentos básicos para o planejamento e a avaliação de programas educativos oferecidos nas instituições de saúde.

Nesse sentido, o Enfermeiro que trabalha no Centro de Material e Esterilização (CME) enfrenta uma série de desafios, principalmente para gerenciar recursos humanos e materiais de forma a aperfeiçoar resultados que atendam à demanda dos diversos setores da instituição. Esse profissional tem como foco manter o controle sobre os processos ali desenvolvidos, contribuindo para a segurança e eficácia no atendimento ao paciente, contribuindo para a qualidade da assistência prestada (Neis \& Gelbcke, 2014).

O processo de formação e atuação em áreas que exige especialização, a exemplo do CME, é uma questão que merece ser amplamente discutida e revista na comunidade acadêmica, visto que ainda persiste um distanciamento entre a formação e a prática profissional, o que implica em despreparo profissional. Lucon et al. (2017) citam que os profissionais de enfermagem sinalizam a necessidade de capacitação para atua essa área do conhecimento, como forma de garantir a qualidade do serviço prestado e manter níveis reduzidos de riscos de agravo à saúde dos usuários, em relação à complexidade dos processos de esterilização, ao alto custo para a aquisição de instrumentos cirúrgicos e equipamentos mais sofisticados, além dos investimentos na qualificação do profissional de CME.

Sumariamente, o CME requer um profissional de saúde capacitado, com conhecimento técnico específico para planejar, coordenar e controlar todo o processo de trabalho na área operacional. Desse modo, atribui-se ao Enfermeiro, desde os primórdios do surgimento da profissão, a função de promover o cuidado dos pacientes, promovendo também o cuidado com os materiais e a organização do ambiente terapêutico (Hoyashi, Rodrigues \& Oliveira, 2015).

Em um parecer técnico do Conselho Regional de Enfermagem (Coren, 2012) em relação a um questionamento sobre as atribuições legais que podem ser desenvolvidas pela equipe de enfermagem no Centro de Materiais e Esterilização, bem como sobre a responsabilidade do Enfermeiro cita que tais funções estão descritas na Resolução da Diretoria Colegiada da Agência Nacional de Vigilância Sanitária (RDC/ANVISA), de 15 de março de 2012, e na Lei do Exercício Profissional de Enfermagem 
n. 7.498/1986, em relação à equipe e à Enfermeira. Isto é, na graduação, o Enfermeiro deve ser preparado para tais atividades, além de assumir todo o processo de gerenciamento do CME nos serviços de saúde.

A referida Resolução preconiza alguns critérios para que o Enfermeiro possa atuar no CME, incluindo algumas características como: responsabilidade; iniciativa; equilíbrio emocional; habilidade no trato com o público e para o trabalho em equipe; capacidade de organização e concentração; facilidade em memorizar especificações e padronizações; bom condicionamento físico e agilidade.

Nesse sentido, ratifica-se a necessidade de um conhecimento específico da equipe de enfermagem para atuar no gerenciamento de CME, uma vez que há uma diversidade de equipamentos, artigos e instrumentos cirúrgicos, bem como o manejo destes. Assim, "constitui-se área do saber da enfermagem, cujo propósito é garantir produtos seguros para a assistência ao paciente" (Lucon et al., 2017, p. 91).

Os apontamentos acima revelam que os profissionais de enfermagem precisam de conhecimentos cruciais para apresentarem qualidade e segurança dos artigos que dispõe a seus usuários. Fica resguardado ao Enfermeiro, a função coordenar as atividades pautadas nos conceitos recentes dentro da obrigatoriedade exigida por legislações à sua equipe que atua no Centro de Materiais e Esterilização.

O gerenciamento do Enfermeiro é que faz o bom funcionamento do CME, uma vez que esse profissional fica responsável pela organização e distribuição dos materiais esterilizados nas demais unidades de atendimento e de internação de pacientes (Silva, 2017). Portando, o papel do Enfermeiro está no desenvolvimento de competências pertinentes à administração do setor, à execução de atividades técnico-assistenciais e à gestão do capital humano.

A rotina do profissional de enfermagem no Centro de Materiais e Esterilização, segundo Motta (2020), tem como principais atividades: 1. Passar nas unidades de enfermagem nos períodos manhã-tarde; 2 . Checar a validade do material estéril e se há material suficiente para atender à demanda da unidade no período; 3. Repor o material usado; 4. Encaminhar todo o material ao centro de material esterilizado; 5 . Controlar a quantidade usada por cada unidade e adequar a reposição do material de acordo com a necessidade de cada unidade.

Em relação à gestão do Centro de Materiais e Esterilização, Costa et al. (2020) elencam as principais atividades de gerenciamento do CME incluindo: 1. Construções de escalas de trabalho; 2. Compra de suprimentos e instrumentos; 3. Agendamento para a manutenção de máquinas e instrumentos; 4. Contato com unidades consumidoras para receberem produtos de saúde sujos e contaminados e entregar produtos processados; 5. Avaliação de indicadores de qualidade de serviço e implementação das atividades de treinamento.

Diante das competências citadas acima pode-se afirmar que a gerência constitui a atividade primordial do Enfermeiro que atua no CME, compreendendo diversas funções, como planejamento, elaboração de instrumentos administrativos e operacionais, administração de recursos materiais e de pessoal e supervisão. Desse modo, o Enfermeiro tem suas atividades concentradas na organização de materiais e pessoal, sendo que os artigos médico-hospitalares processados ou reprocessados constituem-se no seu objeto de trabalho (Gil, Camelo \& Laus, 2013).

Nesse cenário merece também destaque os dizeres de Costa et al. (2020) no qual consideram que o papel dos trabalhadores de enfermagem no CME é moldado pelas funções desse centro nas organizações hospitalares, as percepções de seus trabalhadores sobre a própria atuação e status, bem como as percepções e julgamentos dos trabalhadores de unidades consumidoras a respeito do referido setor, envolvendo o processamento de produtos para a saúde e o gerenciamento.

Com isso, entende-se que o trabalho do Enfermeiro no CME deve ser considerado um cuidado legítimo, por instrumentalizar o cuidado direto, na medida em que ocorre um reconhecimento de que o preparo de materiais é essencial para o cotidiano da prática assistencial da enfermagem. 


\subsection{A importância do Enfermeiro no CME}

Abordar a questão da valorização do profissional de enfermagem no CME requer uma observação desse próprio setor e sua relevância, uma vez que o entendimento da importância do enfermeiro relaciona-se com a importância do próprio CME nas unidades de saúde. Na concepção de Athanázio (2015), o CME é um dos setores das instituições de saúde que é ainda pouco valorizado, com baixa visibilidade entre gestores e profissionais, em muitos casos com inadequações arquitetônicas, localização que não atende as demandas e, muitas vezes, agregado equivocadamente ao centro cirúrgico.

Diante da menção acima, ressalta-se o quão o CME é subvalorizado, embora assume papel de destaque no âmbito dos serviços de saúde, tanto como unidade funcional a todos os serviços de assistência e de diagnóstico, quanto a responsabilidade do processamento de materiais. O desempenho dos profissionais de saúde demonstra a importância desse setor no que tange a responsabilidade no processamento de materiais.

O Conselho Federal de Enfermagem traçou o perfil da enfermagem no Brasil no tocante ao CME e indicou que 80\% dos profissionais que atuam nesse setor são técnicos de enfermagem e 20\% são enfermeiros (Cofen, 2012). A pesquisa mostrou que, na maioria dos casos, o Enfermeiro é quem assume a gerência do CME, contudo, parece não haver uma autonomia nem uma valorização da importância técnico científica de se estar nesta posição. O profissional de CME por vezes experimenta certa invisibilidade, visto que o cuidado que não está diretamente ligado à assistência, ou seja, o cuidado indireto é menos valorizado, apesar de compor um exercício baseado em saberes técnicos e científicos. A qualidade do trabalho do Enfermeiro não se relaciona apenas a sua formação, mas na qualidade estrutural proporcionada pelas instituições onde o profissional desenvolve seus serviços (Parente et al., 2018).

Sanchez et al. (2018) compartilham das ideias acima mencionadas ao afirmarem que o trabalho do Enfermeiro pode se tornar pouco visível diante da ausência de conhecimento acerca do trabalho desenvolvido na CME; da pouca valorização do fazer do enfermeiro da CME, por prestar uma assistência indireta com o paciente; da ausência de critérios de seleção dos funcionários e da alocação de trabalhadores com problemas de saúde e/ou limitações físicas, bem como da rotatividade de funcionários no setor; da ausência de educação permanente voltada para as atividades desenvolvidas na CME; e da pouca divulgação do seu fazer e da sua interdependência com outros setores, ocasionando problemas internos na CME.

Outro fator mencionado por Santos et al. (2017) que compromete a valorização e visibilidade do Enfermeiro que trabalha no CME deve-se a ineficiência da comunicação entre os profissionais atuantes no CME, centro cirúrgico e demais setores das instituições de saúde, tornando prejudicial ao processo de trabalho.

Por esses motivos, a questão da desvalorização do Enfermeiro que atua no CME implica em sérios problemas que podem comprometer o desempenho desse profissional, entre outros, a insatisfação e, consequentemente, a alta rotatividade de pessoal no setor. Ainda, absenteísmo, as constantes licenças médicas, o elevado número de funcionários com depressão e outros agravos de saúde, bem como o sentimento de descontentamento frente à falta de reconhecimento do seu trabalho pelos profissionais das demais unidades hospitalares (Anjos \& Oliveira, 2016). Para Gouveia, Ribeiro \& Carvalho (2020), a satisfação profissional é entendida como medida de qualidade de vida, uma vez que uma pessoa satisfeita é mais produtiva.

Silva (2017) ressalta que o trabalho prescrito de enfermagem no CME se insere nos saberes científicos inerentes à referida profissão. Contudo, o trabalho executado na prática é estigmatizado pela cultura da não visibilidade, influenciando negativamente o profissional que, em determinadas situações, não se posiciona por insegurança em definir e reconhecer seu próprio papel diante de outros profissionais, implicando no adoecimento biopsicossocial do trabalhador do CME.

Gonçalves \& Santana (2016) indicam uma problemática em relação ao trabalho do Enfermeiro no CME, ao revelarem que instituições de saúde e até mesmo gerentes de enfermagem não valorizam a presença do enfermeiro no CME, ficando a cargo dos próprios técnicos e auxiliares de enfermagem a supervisão das atividades. Quando o Enfermeiro está presente, suas atividades não são exclusivas no CME, dividindo suas atenções também com o centro cirúrgico. 
Lucon et al. (2017) ressaltam que grande parte dos hospitais e das unidades de saúde que possui CME insere Enfermeiros para a condução dos seus trabalhos. Portanto, em relação ao gerenciamento, o cargo no CME precisa ser ocupado por um profissional de saúde de nível superior com qualificação específica, experiência na área e que responda legalmente às ações ali realizadas. Acredita-se, assim, que o profissional de enfermagem tem perfil apropriado para realizá-lo.

Nesse sentido, torna-se fundamental a valorização e o incentivo à cultura de organização institucional de modo interativo, flexível, com escuta e foco na satisfação do trabalhador, e também na oferta de materiais e insumos adequados para as suas funções, pois isto certamente irá auxiliá-lo no seu reconhecimento profissional e da sua importância no ambiente de trabalho (Parente et al., 2018).

No CME, a execução das atividades laborativas dos profissionais de enfermagem caracterizam um cuidado legítimo, por instrumentalizar a assistência, cujo reconhecimento de que o preparo de materiais para o cotidiano da prática de enfermagem é essencial, pois a existência de falhas nesses processos é determinante para o surgimento de complicações nos pacientes (Silva, 2017).

Pela complexidade dos trabalhos realizados pelo CME, a atitude de cada colaborador nesse setor e o trabalho de supervisão do Enfermeiro reflete e influencia diretamente na exequibilidade de prática segura ao paciente cirúrgico, mesmo que o cuidado seja indireto, garantindo a reprodutibilidade do processo em sua totalidade. Tais atitudes permeiam a possibilidade do rastreamento de todas as fases do processamento de materiais no que tange ao controle de infecção hospitalar no caso de um necessário recall de produtos para saúde (Moriya \& Takeiti, 2016).

No Brasil, a preocupação com a segurança do paciente tornou-se pauta prioritária no âmbito da saúde, cujo desenvolvimento de estratégias para a segurança do paciente em setores como CME depende do conhecimento e cumprimento do conjunto de normas e regulamentos que regem o funcionamento dos estabelecimentos de saúde.

O RDC n. 15 no qual destaca a atividade de processamento de esterilização deverá ser exercido por profissionais com atividades regulamentadas pelo conselho de classe em consonância com a Resolução n. 424/2012 do COFEN que normatiza as atribuições dos profissionais de enfermagem em Centros de Materiais e Esterilização atribuem ao Enfermeiro a tarefa de realizar as ações de organização, planejamento, orientação e documentação das atividades, enquanto o técnico e auxiliar de enfermagem executar as atividades sob sua supervisão. A RDC n. 15, art. 34, Parágrafo III, o profissional de enfermagem do CME deve ter domínio de toda a logística que abrange o fluxo de solicitação e recebimento de Órtese, Prótese, Materiais Especiais e Síntese (OPMES), e o tratamento correto para o descarte na retirada de material de síntese (explantes) (Cofen, 2012 \& Brasil, 2012).

Com isso, afirma-se que é delegada ao Enfermeiro a chefia do CME deve, em observância à regulamentação da Lei n. 7.498/1986 pelo Decreto-Lei n ${ }^{\circ}$ 94.406/1987, que dispõe sobre o exercício da enfermagem e dá outras providências, cabendo à equipe de enfermagem a responsabilidade no preparo de materiais e equipamentos hospitalares para o uso (Hoyashi, Rodrigues \& Oliveira, 2015).

Conforme observadas antes, as resoluções determinadas pelo Coren (2012), Cofen (2012) \& Brasil (2012) ressaltam a importância da presença de um Enfermeiro qualificado atuando no Centro de Materiais e Esterilização. Gonçalves e Santana (2016) concordam que a exclusividade de um Enfermeiro nesse setor baseia-se em seu conhecimento em relação às ações de cuidado de enfermagem, capacidade de visualização das necessidades do trabalho de outros Enfermeiros e profissionais de saúde que utilizam os produtos do CME, conferindo-lhe assim, características fundamentais à coordenação do setor.

Strieder et al. (2019) complementam as ideias de Gonçalves e Santana (2016), reforçando a importância da atuação exclusiva do Enfermeiro no CME, supervisionando as atividades diárias, padronizando produtos e procedimentos de trabalho, além de elaborar protocolos e orientar de funcionários, o que contribui em uma prática adequada nas etapas de processamento dos produtos de saúde, controle de infecções e prestação de cuidado seguro aos usuários. 
Algumas estratégias podem promover a visibilidade do Enfermeiro em Centro de Materiais e Esterilização, apontam Sanchez et al. (2018), entre eles: a divulgação dos serviços prestados no CME para os demais setores da unidade de saúde, partindo do Enfermeiro em parceria com a educação continuada; a educação continuada/permanente voltada para as temáticas pertinentes ao trabalho da enfermagem desenvolvido na CME compõe uma das estratégias fundamentais para valorização e visibilidade dos profissionais de enfermagem; as relações interpessoais e a comunicação efetiva dos trabalhadores do CME com os demais setores de uma unidade saúde; a implantação de novas tecnologias que possibilitem a monitoração do tempo e rastreabilidade do material, dependendo, contudo, da cooperação de outros serviços para ser implantada.

\subsection{Educação continuada para a equipe de enfermagem que atua no CME}

As atividades do CME têm sua gênese no século XIX, e desde então, acompanham as necessidades dos procedimentos cirúrgicos na perspectiva do controle de infecções. Devido a sua relevância e a grande inserção dos Enfermeiros neste espaço, há a necessidade de uma capacitação contínua no desenvolvimento e no aperfeiçoamento dos recursos humanos deste espaço (Araújo et al., 2016).

Estudos evidenciam que grande parte dos Enfermeiros que estão à frente do CME estimulam os profissionais deste serviço a ingressarem na educação continuada, visto que os profissionais que passam por este processo obtêm melhores resultados em sua prática diária. No entanto, alguns profissionais de enfermagem do CME relatam estar nas atividades de EC de forma obrigatória, não valorizando da forma correta o espaço de construção e crescimento profissional (Araújo et al., 2016).

Implantar programas de educação que abrangem toda a equipe que atua no CME propiciará "transformações no método de trabalho, por meio de sensibilização, envolvimento, compartilhamento e aplicação do conhecimento científico nas práticas diárias" (Morais et al., 2018, p. 67).

A educação continuada requer do Enfermeiro conhecimentos pertinentes à administração, gestão de pessoas, prestação da assistência, no sentido da compreensão da importância da qualidade no processamento de materiais. Para isso são necessários saberes definidos, visto a importância do cumprimento das necessidades exigidas pelo trabalho na CME, direcionando-se à assistência e prática seguras, qualificada aos clientes (Padoveze et al., 2013).

Frente a essa problemática é que a educação continuada ou educação permanente em saúde surge como uma proposta crucial para mudança nos processos de trabalho e suas relações no setor, oferecendo melhorias na assistência de enfermagem, visando o aperfeiçoamento dos profissionais, bem como os espaços de aprendizagem para o próprio setor de trabalho (Ouriques $\&$ Machado, 2013).

Nos estudos exploratórios de Padoveze et al. (2013) a respeito da necessidade de aprendizagem de enfermeiros sobre o processo de esterilização, constatou-se que a opção preferencial da maioria dos Enfermeiros foi pelas modalidades de treinamento na forma de cursos presenciais com aulas teórico-práticas, que abordassem tópicos como: embalagens, métodos de esterilização, controle, monitoramento e validação dos processos de esterilização e noções de microbiologia.

Para Athanázio (2015), a educação continuada funciona como uma estratégia para a organização do processo de trabalho da enfermagem em articulação com as demais práticas de enfermagem e demais setores das instituições de saúde.

Padoveze et al. (2013) afirmam que é bastante recomendado que os Enfermeiros de CME promovam processos educativos formais, visando abranger todas as ações desenvolvidas sistematicamente, no curto, médio, ou longo prazo, que objetiva aumentar a capacitação dos profissionais, visando uma ampliação das atividades profissionais relativas à eficiência na realização das atividades.

As ideias de Parente et al. (2018) corroboram com as de Athanázio (2015) e Padoveze et al. (2013), ao enfatizarem a importância da realização de atividades de educação permanente desenvolvidas na CME, uma vez que possibilita diminuir possíveis falhas ocorridas nos processos de trabalho. Além disso, a capacitação técnica em uma área específica facilita a inserção 
dos trabalhadores e desenvolve a prática profissional no ambiente de trabalho, contribuindo para a reflexão sobre a importância de seu trabalho.

\section{Considerações Finais}

Esta pesquisa teve o intuito de compreender de forma mais profunda a respeito do Centro de Materiais e Esterilização como setor vital de uma instituição de saúde e que o papel do Enfermeiro é essencial para garantir a assistência à saúde segura e eficiente. Permitiu entender, também, que a rotina de trabalho, os parâmetros adequados e recursos humanos para a prática laboral refletem diretamente peculiaridade e qualidade do CME.

Embora seja de conhecimento que algumas unidades de saúde que possuem o Centro de Materiais e Esterilização não dispõe de profissional capacitado nesse setor - o profissional de enfermagem - torna-se difícil em identificar esses locais e acionar autoridades competentes para cumprir o que determina a RDA n. 15/2012 e a resolução do COFEN n. 424/2012 pela insuficiência de estudos que comprovem tal situação.

Por essa questão, as limitações desse estudo devem-se a poucos estudos que contemplasse o objetivo postulado, haja vista grande parte das publicações se concentrarem em riscos físicos e ocupacionais vinculados ao CME. Os poucos estudos que direcionam para a importância do trabalho do Enfermeiro no CME, abordando fatores como carga de trabalho e estratégias de valorização profissional são poucas e restritas a estudo de uma determinada instituição de saúde.

Diante disso, é fundamental que se tenha estudos mais aprofundados, com pesquisas de campo, a fim de se ter um mapeamento detalhado sobre a situação de CME existentes no Brasil, e até a nível regional, que constatasse a presença ou não do Enfermeiro no gerenciamento de atividades, bem como a carga de trabalho, a valorização profissional e realização de educação em saúde continuada, considerada estratégia fundamental no constante aprimoramento do trabalhador.

Com isso, os resultados propiciariam um cenário real da situação dos profissionais de enfermagem em CME no Brasil e, a partir de então, encaminhar aos órgãos competentes para criação de políticas que exija a presença desse profissional no CME com todos os parâmetros necessários para que se realize as atividades permanentes ao setor visando a biossegurança, a saúde e condições de trabalho adequados para o trabalhador, preconizados nas normas regulamentadoras.

\section{Referências}

Alves, H. E., Valença, C. N., Guedes, D. T., Reis, A. C. R., Sugette, J. F. V., \& Cabral, S. A. A. O. (2017). Riscos ocupacionais a que os trabalhadores da enfermagem referem estar expostos em central de material estéril. Id on Line Rev. Psic., 11(37): 1-12.

Anjos, M.A. M., Oliveira, J.C. (2016). As percepções dos profissionais de enfermagem da central de material e esterilização: uma reflexão sobre a cultura organizacional. Revista ACRED, 6(11):1-9.

Aquino, J. M. (2014). Centro de material e esterilização: acidentes de trabalho e riscos ocupacionais. Rev. SOBECC, 19(3):148-54.

Araruna, A. B., \& Posso, M. B. S. (2014). Centro de material de esterilização: parâmetros espaciais e físicos. Rev. SOBECC, 19(3):142-7.

Araújo, L. O., Bezerra, E. N., Lemos, F. S., Silva, M. L. F. S., Silva, J. F. P., \& Silva, T. M. (2016). O papel da enfermagem na educação continuada do centro de material e esterilização. Revista Saúde, 10(1):1.

Athanázio, A. R. (2015). Educação permanente a trabalhadores do Centro de Material e Esterilização: uma contribuição da enfermagem. 114f. Dissertação (Mestrado) - Universidade Federal Fluminense. Niterói: UFF, 2015.

Bittencourt, V. L. L. (2015). Vivências de profissionais de enfermagem sobre riscos ambientais em um centro de material e esterilização. REME - Rev Min Enferm., 19(4): 864-70. 10.5935/1415-2762

Borgheti, S. P., Viegas, K., \& Caregnato, R. C. A. (2016). Biossegurança no centro de materiais e esterilização: dúvidas dos profissionais. Rev. SOBECC, São Paulo, 21(1): 3-12.

Brasil. (2009). Agência Nacional de Vigilância Sanitária. ANVISA. Resolução - RDC No 8, de 27 de fevereiro de 2009. Dispõe sobre as medidas para redução da ocorrência de infecções por Micobactérias de Crescimento Rápido - MCR em serviços de saúde. WWW.portalses.saude.sc.gov.br/index.php?option=com...task. 
Brasil. (2012). Ministério da Saúde. Agência Nacional de Vigilância Sanitária. ANVISA. Resolução - RDC n. 15, de 15 de março de 2012: dispõe sobre requisitos de boas práticas para o processamento de produtos para saúde e dá outras providências. Brasília, 2012.

Bugs, T. V., Rigo, D. F. H., Bohrer, C. D., Borges, F., Marques, L. G., Vasconcelos, R. O., \& Alves, D. C. I. (2017). Perfil da equipe de enfermagem e percepções do trabalho realizado em uma central de materiais. REME - Rev. Min. Enf., 21(1):1-8.

Carvalho, H. E. F., Silva, V. F. M., Silva, D. L., Ribeiro, I. P., Adélia, D. S., \& Madeira, M. Z. A. (2019). Visão dos Profissionais de Enfermagem Quanto aos Riscos ocupacionais e Acidentes de Trabalho na Central de Material e Esterilização. J. res.: fundam. care. online, 11(5):1161-6.

Conselho Federal de Enfermagem - COFEN (2012). Resolução n. 424, de 19 de abril de 2012. Normatiza as atribuições dos profissionais de Enfermagem em Centro de Material e Esterilização e em empresas processadoras de produtos para saúde. 78

Conselho Regional de Enfermagem de São Paulo. (2012). Parecer n. 3, de 5 de julho de 2012: dispõe sobre as atribuições dos profissionais de enfermagem no Centro de Material e Esterilização - CME. São Paulo: Coren-SP, 2012. http://portal.coren-sp.gov.br/sites/default/files/parecer_coren_sp_2012_3.pdf.

Costa, R., Montenegro, H. R. A., Silva, R. N., \& Almeida Filho, A. J. (2020). Papel dos trabalhadores de enfermagem no centro de material e esterilização: revisão integrativa. Escola Anna Nery, 24(3): 1-13.

Costa, C. C. P., Souza, N. V. D. O., Silva, P. A. S., Oliveira, E. B., \& Vieira, M. L. C. (2015). O trabalho na central de material: repercussões para a saúde dos trabalhadores de enfermagem. Rev Enferm UERJ, 23(4):533-9.

Fontana, R. T., \& Lautert, L. (2013). A situação de trabalho da enfermagem e os riscos ocupacionais na perspectiva da ergologia. Rev. Latino-Am. Enfermagem, 21(6):1306-13.

Fusco, S. F. B. \& Spiri, W. C. (2014). Análise dos indicadores de qualidade de centros de material e esterilização de hospitais públicos acreditados. Texto Contexto Enferm, Florianópolis, 23(2): 426-33.

Gil, R. F., Camelo, S. H., \& Laus, A. M. (2013). Atividades do enfermeiro de centro de material e esterilização em instituições hospitalares Texto Contexto Enferm, 22(4): 927-34.

Gonçalves, R. C. S., \& Santana, R. F. (2016). Processo de enfermagem no Centro de Material de Esterilização: revisão integrativa. Academus Revista Científica da Saúde, SMSRIO, 1(2):1-9.

Gouveia, L. H. A., Ribeiro, V. F., \& Carvalho, R. (2020). Satisfação profissional de enfermeiros que atuam no bloco cirúrgico de um hospital de excelência. Rev. SOBECC, 25(1):33-41.

Hoyashi, C. M. T., Rodrigues, D. C. G. A., \& Oliveira, M. F. A. (2015). Central de material e esterilização na formação do Enfermeiro: proposta de um Manual de Práticas. Revista Práxis, 7(14):35-45.

Lima, M. D. P., Chaves, B. J. P., Lima, V. S., Silva, P. E., Soares, N. S. C. S, \& Santos, I. B. C. (2018). Riscos ocupacionais em profissionais da enfermagem de centros de material e esterilização. Revista Cuidarte, 9(3):2361-8.

Lucon, S. M. R., Braccialli, L. A. D., Pirolo, S. M., \& Munhoz, C. C. (2017). Formação do Enfermeiro para atuar na central de esterilização. Rev. SOBECC, 22(2): 90-7.

Madeira, M. Z. A., Santos, A. M. R., Batista, O. M. A., \& Rodrigues, F. T. C. (2015). Processamento de produtos para saúde em centro de material e esterilização. Rev. SOBECC, 20 (4): 220-7.

Marcondes, M. M. S., Montanari, D. C. P. (2020). Esterilização e medidas de biossegurança em Centros de Materiais e Esterilização e outros estabelecimentos. São Paulo: SENAC, 2020.

Marconi, M. A.\& Lakatos, E. M. (2018). Técnicas de pesquisa. (8a ed.), Atlas, 2018.

Martins, J. F., \& Antunes, A. V. (2019). Dimensionamento de pessoal no centro de material e esterilização de um hospital univ ersitário. Rev Esc Enferm USP, São Paulo, p. 1-9, 2019.

Mendes, C. (2017). Avaliação e Controlo de Riscos em Centrais de Esterilização em Contexto Hospitalar. Investigação Aplicada em Saúde Ambiental, 18(1):114.

Moraes, M. V. G. (2012). Enfermagem do trabalho: programas, procedimentos e técnicas. (4a ed.), Iátria, 2012.

Morais, L. M. C., Queiroga, S. S., Santos, A. N., Oliveira, J. M. D., \& Melo, J. T. S. (2018). Processo de esterilização sob a ótica dos profissionais do centro de material e esterilização. Rev. SOBECC, 23(2):61-68.

Ouriques, C. M., \& Machado, M. E. (2013). Enfermagem no processo de esterilização de materiais. Texto Contexto Enferm, 22(3):695-703.

Moriya, G. A. A., \& Takeiti, M. H. (2016). O trabalho da enfermagem em centro de material e esterilização e sua implicação para a segurança do paciente. Rev. SOBECC, 21(1):1-2.

Motta, A. L. C. (2020). Normas, rotinas e técnicas de enfermagem. (7a ed.), Iátria, 2020.

Neis, M. E. B., \& Gelbcke, F. L. (2014). Carga de trabalho em Centro de Material e Esterilização. Rev. SOBECC, 19(1): 11-17.

Padoveze, M. C., Pelaes, C. E. S., Figueiredo, R.M., \& Otrenti, E. (2013). Necessidade de aprendizagem de enfermeiros sobre processo de esterilização. Rev. SOBECC, 18(3): 23-9. 
Research, Society and Development, v. 10, n. 7, e52210713546, 2021

(CC BY 4.0) | ISSN 2525-3409 | DOI: http://dx.doi.org/10.33448/rsd-v10i7.13546

Parente, A. N., Torres, R. S. C., Macedo, W. T. P., Freitas, A. T. M., Silva, C. S. O., \& Silva, E. C. L. (2018). Evidências profissionais de enfermeiros em Central de Material e Esterilização: contribuições de uma revisão integrativa. Open Journal Systems. 17(4):377-85.

Rosa, N.T., Mimura, V. A., \& Borges, E. C. P. (2019). Carga de trabalho e dimensionamento dos profissionais de enfermagem no centro de material e esterilização. Revista nursing, 22(250):2775-82.

Sanchez, M. L., Silveira, R. S., Figueiredo, P. P., Mancia, J. R., Schwonke, C. R. G. B., \& Gonçalves, N. G. C. (2018). Estratégias que contribuem para a visibilidade do trabalho do Enfermeiro na central de material e esterilização. Texto Contexto Enferm, 27(1):1-9.

Santos, L. B. A., Moura, G. L., Araujo, J. D. B., \& Gouveia, M. T. M. (2017). Atuação da equipe de enfermagem em um centro de materiais e esterilização: relato de experiência. In: CONBRACIS: Congresso Brasileiro De Ciências Da Saúde, 2. Campina Grande, 2017. Anais eletrônico...Campina Grande, 2017.

Santos, I. B. C. S. C., Cordeiro, M. F. G. S., Melo, A. C., Lima, V. S., Chaves, B. J. P., \& Silva, P. E. (2017). Equipamentos de proteção individual utilizados por profissionais de enfermagem em centros de material e esterilização. Rev. SOBECC, 22(1): 36-41.

Seibel, L. R. S. (2017). Riscos e desafios enfrentados pelos profissionais no centro de material esterilizado. Trabalho de Conclusão de Curso (Graduação) Faculdade de Ciências da Educação e Saúde - FACES; UniCEUB. UniCEUB, 2017.

Silva, M. B. (2017). A invisibilidade do trabalho de enfermagem do Centro de Materiais e Esterilização. 150f. Dissertação (Mestrado) - Escola de Enfermagem Anna Nery; Universidade Federal do Rio de Janeiro. UFRJ, 2017.

Souza, M. C. B. (2012). O trabalho no centro de material e esterilização. 238 f. Tese (Doutorado) - Faculdade de Ciências Médicas; Universidade Estadual de Campinas. UNICAMP, 2012.

Strieder, A. T., Graube, S. L., Dezordi, C. C.M., Stumm, E. M. F., Meneghete, M. C., \& Bittencourt, V. L. L. (2019). Atuação do enfermeiro no processo de limpeza em um centro de material e esterilização. Rev. SOBECC, 24(1):50-53.

Vital, J. S., Lins, T. H., Veríssimo, R. C. S. S. \& Souza, E. M. S. (2014). Estrutura física de centro de material e esterilização em unidades de atenção básica de saúde. Rev. Enferm UFPE on line, 8(5):1192-1200. 